\title{
Green's function for the wavized Maxwell fish-eye problem
}

\author{
Radosław Szmytkowski \\ Atomic Physics Division, Department of Atomic Physics and Luminescence, \\ Faculty of Applied Physics and Mathematics, Gdańsk University of Technology, \\ Narutowicza 11/12, 80-233 Gdańsk, Poland \\ email: radek@mif.pg.gda.pl \\ Published as: J. Phys. A 44 (2011) 065203 \\ doi: $10.1088 / 1751-8113 / 44 / 6 / 065203$
}

\begin{abstract}
Unique transformation properties under the hyperspherical inversion of a partial differential equation describing a stationary scalar wave in an $N$-dimensional $(N \geqslant 2)$ Maxwell fish-eye medium are exploited to construct a closed form of the Green's function for that equation. For those wave numbers for which the Green's function fails to exist, the generalized Green's function is derived. Prospective physical applications are mentioned.
\end{abstract}

Key words: Maxwell's fish-eye problem; Green's function; Legendre functions; scalar wave optics; gradient-index (GRIN) optics

PACS: 02.30.Jr, 02.30.Gp, 42.25.Bs, 42.79.Ry

MSC: 35J08, 78A10

\section{Introduction}

In 1854, Maxwell [1] pointed at a remarkable property of an infinite optical medium with the refraction index

$$
n_{\mathrm{fe}}(\boldsymbol{r})=\frac{2 n_{0} \rho^{2}}{r^{2}+\rho^{2}} \quad(\rho>0) .
$$

Within the framework of the geometrical optics, he proved that paths of all rays emitted from an arbitrarily located point $\boldsymbol{r}^{\prime}$ are circles having two points in common: the source point $\boldsymbol{r}^{\prime}$ and the image point $-\rho^{2} \boldsymbol{r}^{\prime} / \boldsymbol{r}^{\prime 2}$. For this medium Maxwell coined the name 'the fish-eye'. In 1926, Carathéodory [2] observed that there is a geometrical correspondence between the circular rays in the fish-eye medium and the geodesics on a sphere. This geometric thread was pursued further by other researchers within the group-theoretical framework (cf, e.g., Ref. [3]).

It seems that Demkov and Ostrovsky [4] were the first to discuss the wavized scalar fish-eye problem. Specifically, they considered the equation

$$
\left[\nabla^{2}+\frac{4 \nu(\nu+1) \rho^{2}}{\left(r^{2}+\rho^{2}\right)^{2}}\right] \Psi(\boldsymbol{r})=0 \quad(\rho>0)
$$

in $\mathbb{R}^{3}$, subject to the boundary condition that $\Psi(\boldsymbol{r})$ vanishes at infinity. They proceeded in two directions. First, they solved analytically a spectral problem with $\nu$ being an eigenparameter and showed that the resulting spectrum is purely discrete and eigenfunctions may be expressed in terms of the Gegenbauer polynomials. Second, they proved that Eq. (1.2) possesses a certain remarkable transformation property under the geometrical inversion in a certain class of spheres, 
and ingeniously exploited this fact to construct a closed form of the relevant Green's function in $\mathbb{R}^{3}$. Group-theoretical properties of the scalar fish-eye wave equation (1.2) were then investigated in $\mathbb{R}^{2}$ by Frank et al. $[5,6]$. Lately, the two-dimensional fish-eye medium has been studied by Makowski and Górska [7] in the context of the construction of pertinent coherent states. Finally, in two very recent papers, Leonhardt [8] and Leonhardt and Philbin [9] have argued that the geometric-optical perfect focusing property of the fish-eye medium, discovered by Maxwell, holds as well within the wave-optics framework; that issue will be critically reexamined in our upcoming work, with the aid of the results presented below.

The present paper is the first out of a series of several reports in which we shall expose results of our research on the wave properties of the Maxwell fish-eye and related media. Here, we derive a closed form of the Green's function for the scalar fish-eye wave equation in $\mathbb{R}^{N}, N \geqslant 2$. The particular method we employ generalizes the aforementioned one used by Demkov and Ostrovsky in the case of $N=3$ and exploits a peculiar transformation property of the $N$-dimensional fish-eye equation under the hyperspherical inversion.

The structure of the paper is as follows. In Section 2, we investigate transformation properties of a class of partial differential equations under the hyperspherical inversion, with a special focus on the $N$-dimensional fish-eye equation. The results of that investigation are used in Section 3 to construct the Green's function for the fish-eye problem. The case when the Green's function fails to exist and is to be replaced by the generalized Green's function is considered in Section 4. Prospective physical applications of the results are briefly discussed in Section 5. The paper ends with an appendix, in which a number of closed-form representations of the derivative $\left[\partial P_{\nu}^{-N / 2+1}(x) / \partial \nu\right]_{\nu=n+N / 2-1}$, with $x \in(-1,1), N \in \mathbb{N} \backslash\{0,1\}$ and $n \in \mathbb{N}$, required in Section 4, are displayed. The list of references attached has been intended to contain representative items rather than to be a comprehensive one. An exhaustive listing of works relevant to the Maxwell fish-eye problem will be included in one of our forthcoming papers.

\section{Transformation properties of a class of partial differential equations under the hyperspherical inversion}

At first, we establish the following

Lemma 1. If $\Psi(\boldsymbol{r})$ (with $\boldsymbol{r} \in \mathbb{R}^{N}, N \geqslant 2$ ) satisfies the equation

$$
\left[\nabla^{2}+k^{2} n^{2}(\boldsymbol{r})\right] \Psi(\boldsymbol{r})=0,
$$

then for arbitrary $R \in \mathbb{R}$ and $\boldsymbol{a}, \boldsymbol{b} \in \mathbb{R}^{N}$ it holds that

$$
\left[\nabla^{2}+\frac{k^{2} R^{4}}{|\boldsymbol{r}-\boldsymbol{a}|^{4}} n^{2}\left(\frac{R^{2}}{|\boldsymbol{r}-\boldsymbol{a}|^{2}}(\boldsymbol{r}-\boldsymbol{a})+\boldsymbol{b}\right)\right] \frac{1}{|\boldsymbol{r}-\boldsymbol{a}|^{N-2}} \Psi\left(\frac{R^{2}}{|\boldsymbol{r}-\boldsymbol{a}|^{2}}(\boldsymbol{r}-\boldsymbol{a})+\boldsymbol{b}\right)=0 .
$$

Proof. The transformation

$$
\boldsymbol{r} \mapsto \frac{R^{2}}{|\boldsymbol{r}-\boldsymbol{a}|^{2}}(\boldsymbol{r}-\boldsymbol{a})+\boldsymbol{b}
$$

results in the following alteration of the infinitesimal line element:

$$
(\mathrm{d} \boldsymbol{r})^{2} \mapsto \frac{R^{4}}{|\boldsymbol{r}-\boldsymbol{a}|^{4}}(\mathrm{~d} \boldsymbol{r})^{2} .
$$

Hence, substitution (2.3) implies the following transformation of the $N$-dimensional Laplace operator:

$$
\nabla^{2} \mapsto \frac{|\boldsymbol{r}-\boldsymbol{a}|^{2 N}}{R^{2 N}} \nabla \cdot\left(\frac{R^{2(N-2)}}{|\boldsymbol{r}-\boldsymbol{a}|^{2(N-2)}} \nabla\right)
$$

Using the easily provable differential identity

$$
\boldsymbol{\nabla} \cdot\left(\frac{R^{2(N-2)}}{|\boldsymbol{r}-\boldsymbol{a}|^{2(N-2)}} \nabla\right)=\frac{R^{2(N-2)}}{|\boldsymbol{r}-\boldsymbol{a}|^{N-2}} \nabla^{2} \frac{1}{|\boldsymbol{r}-\boldsymbol{a}|^{N-2}}-\frac{R^{2(N-2)}}{|\boldsymbol{r}-\boldsymbol{a}|^{N-2}}\left(\nabla^{2} \frac{1}{|\boldsymbol{r}-\boldsymbol{a}|^{N-2}}\right),
$$


and exploiting the fact that the function $1 /|\boldsymbol{r}-\boldsymbol{a}|^{N-2}$ is harmonic in $\mathbb{R}^{N}$, we see that transformation (2.3) changes Eq. (2.1) into

$$
\left[\frac{|\boldsymbol{r}-\boldsymbol{a}|^{N+2}}{R^{4}} \nabla^{2} \frac{1}{|\boldsymbol{r}-\boldsymbol{a}|^{N-2}}+k^{2} n^{2}\left(\frac{R^{2}}{|\boldsymbol{r}-\boldsymbol{a}|^{2}}(\boldsymbol{r}-\boldsymbol{a})+\boldsymbol{b}\right)\right] \Psi\left(\frac{R^{2}}{|\boldsymbol{r}-\boldsymbol{a}|^{2}}(\boldsymbol{r}-\boldsymbol{a})+\boldsymbol{b}\right)=0
$$

which immediately leads to Eq. (2.2).

Actually, the above lemma offers a bit more than necessary for the purposes of this paper. In view of our needs, in what follows we shall restrict ourselves to the special case when the vectors $\boldsymbol{a}$ and $\boldsymbol{b}$ are equal. It is then evident that the resulting transformation

$$
\boldsymbol{r} \mapsto \frac{R^{2}}{|\boldsymbol{r}-\boldsymbol{a}|^{2}}(\boldsymbol{r}-\boldsymbol{a})+\boldsymbol{a}
$$

is the geometric inversion in the hypersphere of radius $R$ centered at the point with the radius vector $\boldsymbol{r}=\boldsymbol{a}$. (In fact, if $\boldsymbol{b}=\boldsymbol{a}$ and $k=0$, the lemma is simply an $N$-dimensional extension of the well-known Kelvin inversion theorem for harmonic functions [10].) $(2.8)$ :

Now we turn to the fish-eye problem. Application of the following special case of inversion

$$
\boldsymbol{r} \mapsto \frac{\rho^{2}+a^{2}}{|\boldsymbol{r}-\boldsymbol{a}|^{2}}(\boldsymbol{r}-\boldsymbol{a})+\boldsymbol{a} \quad \Rightarrow \quad r \mapsto \frac{a}{|\boldsymbol{r}-\boldsymbol{a}|}\left|\boldsymbol{r}+\boldsymbol{a} \frac{\rho^{2}}{a^{2}}\right|
$$

to the fish-eye refraction index (1.1) gives

$$
n_{\mathrm{fe}}\left(\frac{\rho^{2}+a^{2}}{|\boldsymbol{r}-\boldsymbol{a}|^{2}}(\boldsymbol{r}-\boldsymbol{a})+\boldsymbol{a}\right)=\frac{|\boldsymbol{r}-\boldsymbol{a}|^{2}}{\rho^{2}+a^{2}} \frac{2 n_{0} \rho^{2}}{r^{2}+\rho^{2}}=\frac{|\boldsymbol{r}-\boldsymbol{a}|^{2}}{\rho^{2}+a^{2}} n_{\mathrm{fe}}(\boldsymbol{r}) .
$$

Combining this property of the index $n_{\mathrm{fe}}(\boldsymbol{r})$ with the result stated in the lemma, we arrive at

Corollary. If the function $\Psi(\boldsymbol{r})$ (with $\boldsymbol{r} \in \mathbb{R}^{N}, N \geqslant 2$ ) solves the fish-eye equation

$$
\left[\nabla^{2}+\frac{4 n_{0}^{2} k^{2} \rho^{4}}{\left(r^{2}+\rho^{2}\right)^{2}}\right] \Psi(\boldsymbol{r})=0 \quad(\rho>0),
$$

then for arbitrary $\boldsymbol{a} \in \mathbb{R}^{N}$ the function

$$
\hat{\mathcal{I}}\left(\boldsymbol{a}, \sqrt{\rho^{2}+a^{2}}\right) \Psi(\boldsymbol{r}) \equiv \frac{1}{|\boldsymbol{r}-\boldsymbol{a}|^{N-2}} \Psi\left(\frac{\rho^{2}+a^{2}}{|\boldsymbol{r}-\boldsymbol{a}|^{2}}(\boldsymbol{r}-\boldsymbol{a})+\boldsymbol{a}\right)
$$

also solves this equation, i.e., it holds that

$$
\left[\nabla^{2}+\frac{4 n_{0}^{2} k^{2} \rho^{4}}{\left(r^{2}+\rho^{2}\right)^{2}}\right] \frac{1}{|\boldsymbol{r}-\boldsymbol{a}|^{N-2}} \Psi\left(\frac{\rho^{2}+a^{2}}{|\boldsymbol{r}-\boldsymbol{a}|^{2}}(\boldsymbol{r}-\boldsymbol{a})+\boldsymbol{a}\right)=0 \quad(\rho>0) .
$$

In the particular case of $N=3$, the above result was established by Demkov and Ostrovsky [4] (see also [11]).

\section{The Green's function for the fish-eye problem}

We are now ready to construct the $N$-dimensional fish-eye Green's function $G_{\nu}\left(\boldsymbol{r}, \boldsymbol{r}^{\prime}\right)$. According to the general theory of Green's functions for elliptic partial differential operators, $G_{\nu}\left(\boldsymbol{r}, \boldsymbol{r}^{\prime}\right)$ is a single-valued solution to the fish-eye equation

$$
\left[\nabla^{2}+\frac{4 n_{0}^{2} k^{2} \rho^{4}}{\left(r^{2}+\rho^{2}\right)^{2}}\right] G_{\nu}\left(\boldsymbol{r}, \boldsymbol{r}^{\prime}\right)=0 \quad(\rho>0)
$$


everywhere in $\mathbb{R}^{N}$ except for the source point $\boldsymbol{r}=\boldsymbol{r}^{\prime}$, where it diverges according to

$$
G_{\nu}\left(\boldsymbol{r}, \boldsymbol{r}^{\prime}\right) \stackrel{\boldsymbol{r} \rightarrow \boldsymbol{r}^{\prime}}{\longrightarrow} \frac{1}{2 \pi} \ln \left|\boldsymbol{r}-\boldsymbol{r}^{\prime}\right| \quad(N=2)
$$

or

$$
G_{\nu}\left(\boldsymbol{r}, \boldsymbol{r}^{\prime}\right) \stackrel{\boldsymbol{r} \rightarrow \boldsymbol{r}^{\prime}}{\longrightarrow}-\frac{1}{(N-2) S_{N-1}\left|\boldsymbol{r}-\boldsymbol{r}^{\prime}\right|^{N-2}} \quad(N \geqslant 3) .
$$

In the last equation

$$
S_{N-1}=\frac{2 \pi^{N / 2}}{\Gamma\left(\frac{N}{2}\right)}
$$

is a surface area of a unit $(N-1)$-dimensional sphere $\mathbb{S}^{N-1}$ embedded in $\mathbb{R}^{N}$. At infinity, we require

$$
G_{\nu}\left(\boldsymbol{r}, \boldsymbol{r}^{\prime}\right) \stackrel{r \rightarrow \infty}{\longrightarrow} \frac{C_{\nu}\left(\boldsymbol{r}^{\prime}\right)}{r^{N-2}}
$$

(the non-zero constant $C_{\nu}\left(\boldsymbol{r}^{\prime}\right)$ appearing in condition (3.5) will be determined later). The parameter $\nu$ is defined as

$$
\nu=\frac{-1+\sqrt{1+4 n_{0}^{2} k^{2} \rho^{2}}}{2} \quad\left(\nu \rightarrow 0 \text { for } n_{0} \rightarrow 0\right)
$$

and reasons for its introduction will become clear shortly. In what follows, we admit that the product $n_{0}^{2} k^{2}$, hence also $\nu$, may be complex.

At first, consider the case when the source is located at the center of symmetry of the medium. Evidently, the corresponding Green's function $G_{\nu}(\boldsymbol{r}, \mathbf{0})$ must be spherically symmetric, being a function of $r=|\boldsymbol{r}|$ only. Hence, it follows that $G_{\nu}(\boldsymbol{r}, \mathbf{0})$ obeys

$$
\left[\frac{\partial^{2}}{\partial r^{2}}+\frac{N-1}{r} \frac{\partial}{\partial r}+\frac{4 \nu(\nu+1) \rho^{2}}{\left(r^{2}+\rho^{2}\right)^{2}}\right] G_{\nu}(\boldsymbol{r}, \mathbf{0})=0
$$

except for the point $\boldsymbol{r}=\mathbf{0}$, where it behaves according to Eqs. (3.2) or (3.3) with $\boldsymbol{r}^{\prime}=\mathbf{0}$. The substitution

$$
G_{\nu}(\boldsymbol{r}, \mathbf{0})=\left(\frac{\rho}{r}\right)^{N / 2-1} F\left(\frac{r^{2}-\rho^{2}}{r^{2}+\rho^{2}}\right)
$$

leads to the following differential equation for the function $F$ :

$$
\left[\left(1-x^{2}\right) \frac{\mathrm{d}^{2}}{\mathrm{~d} x^{2}}-2 x \frac{\mathrm{d}}{\mathrm{d} x}+\nu(\nu+1)-\frac{\mu^{2}}{1-x^{2}}\right] F(x)=0,
$$

where

$$
x=\frac{r^{2}-\rho^{2}}{r^{2}+\rho^{2}} \quad(-1 \leqslant x \leqslant 1)
$$

and

$$
\mu=\frac{N-2}{2} .
$$

Equation (3.9) is the associated Legendre equation. Its general solution, written in the form most suitable for the present purposes, is

$$
F(x)=A P_{\nu}^{-\mu}(x)+B R_{\nu}^{\mu}(x),
$$

with $A, B$ being arbitrary constants and with

$$
R_{\nu}^{\mu}(x)=Q_{\nu}^{\mu}(x)+\frac{\mathrm{i} \pi}{2} P_{\nu}^{\mu}(x)=\frac{\pi}{2 \sin (\pi \mu)}\left[\mathrm{e}^{\mathrm{i} \pi \mu} P_{\nu}^{\mu}(x)-\frac{\Gamma(\nu+\mu+1)}{\Gamma(\nu-\mu+1)} P_{\nu}^{-\mu}(x)\right] .
$$

Here, $P_{\nu}^{\mu}(x)$ and $Q_{\nu}^{\mu}(x)$ are the associated Legendre functions (on the cut $-1 \leqslant x \leqslant 1$ ) of the first and second kinds, respectively (occasionally, $R_{\nu}^{\mu}(x)$ is called the associated Legendre function of 
the third kind). The general character of solution (3.12) follows from the fact that the Wronskian of $P_{\nu}^{-\mu}(x)$ and $R_{\nu}^{\mu}(x)$ is

$$
W\left[P_{\nu}^{-\mu}(x), R_{\nu}^{\mu}(x)\right]=\frac{\exp (\mathrm{i} \pi \mu)}{1-x^{2}},
$$

i.e., it vanishes nowhere. Henceforth, we shall adopt the standard convention and shall write $P_{\nu}(x)$ and $R_{\nu}(x)$ in place of $P_{\nu}^{0}(x)$ and $R_{\nu}^{0}(x)$.

With the general solution to Eq. (3.9) in hand, we see that the Green's function $G_{\nu}(\boldsymbol{r}, \mathbf{0})$ is of the form

$$
G_{\nu}(\boldsymbol{r}, \mathbf{0})=A\left(\frac{\rho}{r}\right)^{N / 2-1} P_{\nu}^{-N / 2+1}\left(\frac{r^{2}-\rho^{2}}{r^{2}+\rho^{2}}\right)+B\left(\frac{\rho}{r}\right)^{N / 2-1} R_{\nu}^{N / 2-1}\left(\frac{r^{2}-\rho^{2}}{r^{2}+\rho^{2}}\right),
$$

$\nu$ being defined in Eq. (3.6). We shall fix values of the constants $A$ and $B$ in two steps. At first, we investigate the asymptotics of the expression in Eq. (3.15) as $r \rightarrow \infty$. Using the known formulas $[12$, p. 196$]$

$$
\begin{gathered}
P_{\nu}^{-\mu}(x) \stackrel{x \rightarrow 1-0}{\longrightarrow} \frac{1}{\Gamma(\mu+1)}\left(\frac{1-x}{2}\right)^{\mu / 2} \quad(\mu \neq-1,-2, \ldots), \\
R_{\nu}(x) \stackrel{x \rightarrow 1-0}{\longrightarrow}-\frac{1}{2} \ln (1-x), \\
R_{\nu}^{\mu}(x) \stackrel{x \rightarrow 1-0}{\longrightarrow} \frac{1}{2} \mathrm{e}^{\mathrm{i} \pi \mu} \Gamma(\mu)\left(\frac{1-x}{2}\right)^{-\mu / 2} \quad(\operatorname{Re} \mu>0),
\end{gathered}
$$

we see that the constraint (3.5) is fulfilled iff $B=0$, and consequently

$$
G_{\nu}(\boldsymbol{r}, \mathbf{0})=A\left(\frac{\rho}{r}\right)^{N / 2-1} P_{\nu}^{-N / 2+1}\left(\frac{r^{2}-\rho^{2}}{r^{2}+\rho^{2}}\right)
$$

In the second step, we investigate the asymptotics of the right-hand side of Eq. (3.19) as $\boldsymbol{r} \rightarrow \mathbf{0}$. Exploiting the formulas [12, p. 197]

$$
P_{\nu}(x) \stackrel{x \rightarrow-1+0}{\longrightarrow} \frac{\sin (\pi \nu)}{\pi} \ln (1+x)
$$

and

$$
P_{\nu}^{-\mu}(x) \stackrel{x \rightarrow-1+0}{\longrightarrow} \frac{\Gamma(\mu)}{\Gamma(\nu+\mu+1) \Gamma(-\nu+\mu)}\left(\frac{1+x}{2}\right)^{-\mu / 2} \quad(\operatorname{Re} \mu>0),
$$

we find that the constraints (3.2) and (3.3) will be satisfied iff

$$
G_{\nu}(\boldsymbol{r}, \mathbf{0})=\frac{1}{4 \sin (\pi \nu)} P_{\nu}\left(\frac{r^{2}-\rho^{2}}{r^{2}+\rho^{2}}\right) \quad(N=2)
$$

and

$$
G_{\nu}(\boldsymbol{r}, \mathbf{0})=-\frac{\Gamma\left(\frac{N}{2}+\nu\right) \Gamma\left(\frac{N}{2}-\nu-1\right)}{4 \pi^{N / 2}} \frac{P_{\nu}^{-N / 2+1}\left(\frac{r^{2}-\rho^{2}}{r^{2}+\rho^{2}}\right)}{(r \rho)^{N / 2-1}} \quad(N \geqslant 3),
$$

respectively. Since it holds that

$$
\sin (\pi \nu)=-\frac{\pi}{\Gamma(\nu+1) \Gamma(-\nu)}
$$

Eqs. (3.22) and (3.23) may be collected into a single formula

$$
G_{\nu}(\boldsymbol{r}, \mathbf{0})=-\frac{\Gamma\left(\frac{N}{2}+\nu\right) \Gamma\left(\frac{N}{2}-\nu-1\right)}{4 \pi^{N / 2}} \frac{P_{\nu}^{-N / 2+1}\left(\frac{r^{2}-\rho^{2}}{r^{2}+\rho^{2}}\right)}{(r \rho)^{N / 2-1}}
$$


From this, using relation (3.16), we deduce that for $\boldsymbol{r}^{\prime}=\mathbf{0}$ the constant $C_{\nu}$ in the asymptotic relation $(3.5)$ is

$$
C_{\nu}(\mathbf{0})=-\frac{\Gamma\left(\frac{N}{2}+\nu\right) \Gamma\left(\frac{N}{2}-\nu-1\right)}{4 \pi^{N / 2} \Gamma\left(\frac{N}{2}\right)} .
$$

To find the Green's function for an arbitrary location of the source point $\boldsymbol{r}^{\prime}$, we consider the transformed function

$$
\hat{\mathcal{I}}\left(\boldsymbol{a}, \sqrt{\rho^{2}+a^{2}}\right) G_{\nu}(\boldsymbol{r}, \mathbf{0})=\frac{1}{|\boldsymbol{r}-\boldsymbol{a}|^{N-2}} G_{\nu}\left(\frac{\rho^{2}+a^{2}}{|\boldsymbol{r}-\boldsymbol{a}|^{2}}(\boldsymbol{r}-\boldsymbol{a})+\boldsymbol{a}, \mathbf{0}\right),
$$

with the center of the inversion sphere (of radius $\sqrt{\rho^{2}+a^{2}}$ ) located at the point

$$
\boldsymbol{a}=-\boldsymbol{r}^{\prime} \frac{\rho^{2}}{r^{\prime 2}}
$$

Using Eq. (3.25), the explicit form of this transformed function, denoted hereafter as $g_{\nu}\left(\boldsymbol{r}, \boldsymbol{r}^{\prime}\right)$, is seen to be

$$
g_{\nu}\left(\boldsymbol{r}, \boldsymbol{r}^{\prime}\right)=-\frac{\Gamma\left(\frac{N}{2}+\nu\right) \Gamma\left(\frac{N}{2}-\nu-1\right)}{4 \pi^{N / 2}}\left(\frac{r^{\prime}}{\rho^{3}}\right)^{N / 2-1} \frac{P_{\nu}^{-N / 2+1}\left(-1+\frac{2 \rho^{2}\left(\boldsymbol{r}-\boldsymbol{r}^{\prime}\right)^{2}}{\left(r^{2}+\rho^{2}\right)\left(r^{\prime 2}+\rho^{2}\right)}\right)}{\left|\boldsymbol{r}-\boldsymbol{r}^{\prime}\right|^{N / 2-1}\left|\boldsymbol{r}+\boldsymbol{r}^{\prime} \frac{\rho^{2}}{r^{\prime 2}}\right|^{N / 2-1}} .
$$

In view of the results of Section 2, we know for sure that the function in Eq. (3.29) solves the fish-eye equation, except, possibly, for some isolated points. It is evident that the points at which the behavior of $g_{\nu}\left(\boldsymbol{r}, \boldsymbol{r}^{\prime}\right)$ should be investigated are the two finite points $\boldsymbol{r}=\boldsymbol{r}^{\prime}$ and $\boldsymbol{r}=-\boldsymbol{r}^{\prime} \rho^{2} / r^{\prime 2}$, and also the point at infinity. Using the asymptotic relations (3.20) and (3.21), we derive

$$
g_{\nu}\left(\boldsymbol{r}, \boldsymbol{r}^{\prime}\right) \stackrel{\boldsymbol{r} \rightarrow \boldsymbol{r}^{\prime}}{\longrightarrow} \frac{1}{2 \pi} \ln \left|\boldsymbol{r}-\boldsymbol{r}^{\prime}\right| \quad(N=2)
$$

and

$$
g_{\nu}\left(\boldsymbol{r}, \boldsymbol{r}^{\prime}\right) \stackrel{\boldsymbol{r} \rightarrow \boldsymbol{r}^{\prime}}{\longrightarrow}-\frac{1}{(N-2) S_{N-1}\left|\boldsymbol{r}-\boldsymbol{r}^{\prime}\right|^{N-2}}\left(\frac{r^{\prime}}{\rho^{2}}\right)^{N-2} \quad(N \geqslant 3),
$$

i.e., the 'inverted' function diverges for $\boldsymbol{r} \rightarrow \boldsymbol{r}^{\prime}$ in the same manner (save for the factor $\left(r^{\prime} / \rho^{2}\right)^{N-2}$ when $N \geqslant 3$ ) as the Green's function $G_{\nu}\left(\boldsymbol{r}, \boldsymbol{r}^{\prime}\right)$ (cf. Eqs. (3.2) and (3.3)). Furthermore, it is seen that for $r \rightarrow \infty$ the function (3.29) decays asymptotically as

$$
g_{\nu}\left(\boldsymbol{r}, \boldsymbol{r}^{\prime}\right) \stackrel{r \rightarrow \infty}{\longrightarrow}-\frac{\Gamma\left(\frac{N}{2}+\nu\right) \Gamma\left(\frac{N}{2}-\nu-1\right)}{4 \pi^{N / 2}}\left(\frac{r^{\prime}}{\rho^{3}}\right)^{N / 2-1} \frac{P_{\nu}^{-N / 2+1}\left(\frac{\rho^{2}-r^{\prime 2}}{\rho^{2}+r^{\prime 2}}\right)}{r^{N-2}},
$$

i.e., in the same functional manner with $r$ as prescribed for $G_{\nu}\left(\boldsymbol{r}, \boldsymbol{r}^{\prime}\right)$ in Eq. (3.5). Finally, with the help of the identity

$$
-1+\frac{2 \rho^{2}\left(\boldsymbol{r}-\boldsymbol{r}^{\prime}\right)^{2}}{\left(r^{2}+\rho^{2}\right)\left(r^{\prime 2}+\rho^{2}\right)}=1-\frac{2 r^{\prime 2}\left(\boldsymbol{r}+\boldsymbol{r}^{\prime} \frac{\rho^{2}}{r^{\prime 2}}\right)^{2}}{\left(r^{2}+\rho^{2}\right)\left(r^{\prime 2}+\rho^{2}\right)}
$$

and the asymptotic relation (3.16), we find that for $\boldsymbol{r} \rightarrow-\boldsymbol{r}^{\prime} \rho^{2} / r^{2}$ the function (3.29) remains finite:

$$
g_{\nu}\left(\boldsymbol{r}, \boldsymbol{r}^{\prime}\right) \stackrel{\boldsymbol{r} \rightarrow-\boldsymbol{r}^{\prime} \rho^{2} / r^{\prime 2}}{\longrightarrow}-\frac{\Gamma\left(\frac{N}{2}+\nu\right) \Gamma\left(\frac{N}{2}-\nu-1\right)}{4 \pi^{N / 2} \Gamma\left(\frac{N}{2}\right)}\left(\frac{r^{\prime}}{\rho}\right)^{2(N-2)} \frac{1}{\left(r^{\prime 2}+\rho^{2}\right)^{N-2}} .
$$


Thus, we see that the function $\left(\rho^{2} / r^{\prime}\right)^{N-2} g_{\nu}\left(\boldsymbol{r}, \boldsymbol{r}^{\prime}\right)$ satisfies all conditions imposed on $G_{\nu}\left(\boldsymbol{r}, \boldsymbol{r}^{\prime}\right)$ in Eqs. (3.1)-(3.5). Hence, we conclude that the closed form of the $N$-dimensional fish-eye Green's function for an arbitrary location of the source point $\boldsymbol{r}^{\prime}$ is

$$
G_{\nu}\left(\boldsymbol{r}, \boldsymbol{r}^{\prime}\right)=-\frac{\Gamma\left(\frac{N}{2}+\nu\right) \Gamma\left(\frac{N}{2}-\nu-1\right)}{4 \pi^{N / 2}}\left(\frac{\rho}{r^{\prime}}\right)^{N / 2-1} \frac{P_{\nu}^{-N / 2+1}\left(-1+\frac{2 \rho^{2}\left(\boldsymbol{r}-\boldsymbol{r}^{\prime}\right)^{2}}{\left(r^{2}+\rho^{2}\right)\left(r^{\prime 2}+\rho^{2}\right)}\right)}{\left|\boldsymbol{r}-\boldsymbol{r}^{\prime}\right|^{N / 2-1}\left|\boldsymbol{r}+\boldsymbol{r}^{\prime} \frac{\rho^{2}}{r^{\prime 2}}\right|^{N / 2-1}}
$$

and that the constant $C_{\nu}\left(\boldsymbol{r}^{\prime}\right)$ in the asymptotic constraint (3.5) is

$$
C_{\nu}\left(\boldsymbol{r}^{\prime}\right)=-\frac{\Gamma\left(\frac{N}{2}+\nu\right) \Gamma\left(\frac{N}{2}-\nu-1\right)}{4 \pi^{N / 2}}\left(\frac{\rho}{r^{\prime}}\right)^{N / 2-1} P_{\nu}^{-N / 2+1}\left(\frac{\rho^{2}-r^{\prime 2}}{\rho^{2}+r^{\prime 2}}\right) .
$$

Since the differential operator in Eq. (3.1) is symmetric with respect to the scalar product $\langle\chi \mid \phi\rangle_{N} \equiv \int_{\mathbb{R}^{N}} \mathrm{~d}^{N} \boldsymbol{r} \chi(\boldsymbol{r}) \phi(\boldsymbol{r})$, the fish-eye Green's function should be symmetric with respect to the interchange of the source and observation points:

$$
G_{\nu}\left(\boldsymbol{r}, \boldsymbol{r}^{\prime}\right)=G_{\nu}\left(\boldsymbol{r}^{\prime}, \boldsymbol{r}\right)
$$

However, the representation of $G_{\nu}\left(\boldsymbol{r}, \boldsymbol{r}^{\prime}\right)$ given in Eq. (3.35) does not exhibit this property explicitly. To show that nevertheless relation (3.37) is satisfied, we observe that it holds that

$$
r^{\prime}\left|\boldsymbol{r}+\boldsymbol{r}^{\prime} \frac{\rho^{2}}{r^{\prime 2}}\right|=r\left|\boldsymbol{r}^{\prime}+\boldsymbol{r} \frac{\rho^{2}}{r^{2}}\right|=\sqrt{r^{2} r^{\prime 2}+2 \rho^{2} \boldsymbol{r} \cdot \boldsymbol{r}^{\prime}+\rho^{4}}
$$

Consequently, the Green's function (3.35) may be alternatively rewritten in either of the following two manifestly symmetric forms:

$$
G_{\nu}\left(\boldsymbol{r}, \boldsymbol{r}^{\prime}\right)=-\frac{\Gamma\left(\frac{N}{2}+\nu\right) \Gamma\left(\frac{N}{2}-\nu-1\right)}{4 \pi^{N / 2}} \frac{\rho^{N / 2-1} P_{\nu}^{-N / 2+1}\left(-1+\frac{2 \rho^{2}\left(\boldsymbol{r}-\boldsymbol{r}^{\prime}\right)^{2}}{\left(r^{2}+\rho^{2}\right)\left(r^{\prime 2}+\rho^{2}\right)}\right)}{\left|\boldsymbol{r}-\boldsymbol{r}^{\prime}\right|^{N / 2-1}\left(r^{2} r^{\prime 2}+2 \rho^{2} \boldsymbol{r} \cdot \boldsymbol{r}^{\prime}+\rho^{4}\right)^{N / 4-1 / 2}}
$$

or

$$
\begin{aligned}
G_{\nu}\left(\boldsymbol{r}, \boldsymbol{r}^{\prime}\right)= & -\frac{\Gamma\left(\frac{N}{2}+\nu\right) \Gamma\left(\frac{N}{2}-\nu-1\right)}{4 \pi^{N / 2}} \\
& \times \frac{\rho^{N / 2-1} P_{\nu}^{-N / 2+1}\left(-1+\frac{2 \rho^{2}\left(\boldsymbol{r}-\boldsymbol{r}^{\prime}\right)^{2}}{\left(r^{2}+\rho^{2}\right)\left(r^{\prime 2}+\rho^{2}\right)}\right)}{\left(r r^{\prime}\right)^{N / 4-1 / 2}\left|\boldsymbol{r}-\boldsymbol{r}^{\prime}\right|^{N / 2-1}\left|\boldsymbol{r}+\boldsymbol{r}^{\prime} \frac{\rho^{2}}{r^{\prime 2}}\right|^{N / 4-1 / 2}\left|\boldsymbol{r}^{\prime}+\boldsymbol{r} \frac{\rho^{2}}{r^{2}}\right|^{N / 4-1 / 2}} .
\end{aligned}
$$

Still another representation of $G_{\nu}\left(\boldsymbol{r}, \boldsymbol{r}^{\prime}\right)$ displaying its symmetry is the one in terms of the Gegenbauer function of the first kind. Using the known relationship

$$
C_{\alpha}^{\lambda}(x)=\frac{\sqrt{\pi}}{2^{\lambda-1 / 2}} \frac{\Gamma(\alpha+2 \lambda)}{\Gamma(\lambda) \Gamma(\alpha+1)}\left(1-x^{2}\right)^{-\lambda / 2+1 / 4} P_{\alpha+\lambda-1 / 2}^{-\lambda+1 / 2}(x) \quad(-1 \leqslant x \leqslant 1),
$$

Eq. (3.35) is transformed into

$$
G_{\nu}\left(\boldsymbol{r}, \boldsymbol{r}^{\prime}\right)=\frac{2^{N-4} \Gamma\left(\frac{N-1}{2}\right)}{\pi^{(N-1) / 2} \sin \left[\pi\left(\frac{N}{2}-\nu\right)\right]} \frac{\rho^{N-2} C_{\nu-N / 2+1}^{(N-1) / 2}\left(-1+\frac{2 \rho^{2}\left(\boldsymbol{r}-\boldsymbol{r}^{\prime}\right)^{2}}{\left(r^{2}+\rho^{2}\right)\left(r^{\prime 2}+\rho^{2}\right)}\right)}{\left(r^{2}+\rho^{2}\right)^{N / 2-1}\left(r^{\prime 2}+\rho^{2}\right)^{N / 2-1}} .
$$

Let us consider some particular cases. For $N=2$, from either of Eqs. (3.35), (3.39) or (3.40), with the aid of Eq. (3.24), we find

$$
G_{\nu}\left(\boldsymbol{r}, \boldsymbol{r}^{\prime}\right)=\frac{1}{4 \sin (\pi \nu)} P_{\nu}\left(-1+\frac{2 \rho^{2}\left(\boldsymbol{r}-\boldsymbol{r}^{\prime}\right)^{2}}{\left(r^{2}+\rho^{2}\right)\left(r^{2}+\rho^{2}\right)}\right) \quad(N=2) .
$$


Next, it appears that the representations of $G_{\nu}\left(\boldsymbol{r}, \boldsymbol{r}^{\prime}\right)$ found above simplify greatly when $N$ is odd, as then the Legendre function appearing in Eqs. (3.35), (3.39) and (3.40) may be expressed in terms of trigonometric and inverse trigonometric functions either as [12, p. 168]

$$
\begin{aligned}
P_{\nu}^{-N / 2+1}(x)= & \frac{\left(\frac{N-3}{2}\right) !}{2^{N / 2-2} \sqrt{\pi}}\left(1-x^{2}\right)^{-N / 4+1 / 2} \sum_{k=0}^{(N-3) / 2}(-)^{k} \frac{\Gamma\left(k+\nu-\frac{N}{2}+2\right)}{k ! \Gamma\left(k+\nu+\frac{3}{2}\right)\left(\frac{N-3}{2}-k\right) !} \\
& \times \sin \left[\left(2 k+\nu-\frac{N}{2}+2\right) \arccos x\right] \quad(N \text { odd, } N \geqslant 3)
\end{aligned}
$$

or as $[12$, p. 169$]$

$$
\begin{aligned}
P_{\nu}^{-N / 2+1}(x)= & \sqrt{\frac{2}{\pi}} \Gamma\left(\nu-\frac{N}{2}+2\right) \sum_{k=0}^{(N-3) / 2} \frac{\left(k+\frac{N-3}{2}\right) !}{2^{k} k ! \Gamma\left(k+\nu+\frac{3}{2}\right)\left(\frac{N-3}{2}-k\right) !} \\
& \times \frac{\sin \left[\left(k+\nu+\frac{1}{2}\right) \arccos x+\left(k-\frac{N-3}{2}\right) \frac{\pi}{2}\right]}{\left(1-x^{2}\right)^{k / 2+1 / 4} \quad(N \text { odd }, N \geqslant 3) .} .
\end{aligned}
$$

In the simplest case of $N=3$, we have

$$
P_{\nu}^{-1 / 2}(x)=\sqrt{\frac{2}{\pi}}\left(1-x^{2}\right)^{-1 / 4} \frac{\sin \left[\left(\nu+\frac{1}{2}\right) \arccos x\right]}{\nu+\frac{1}{2}} .
$$

Using this representation of $P_{\nu}^{-1 / 2}(x)$ in Eq. (3.39), the latter being specialized to the case $N=3$, after some straightforward movements and with the help of the identity

$$
\Gamma\left(\frac{1}{2}+\nu\right) \Gamma\left(\frac{1}{2}-\nu\right)=\frac{\pi}{\cos (\pi \nu)}
$$

we arrive at

$$
\begin{aligned}
G_{\nu}\left(\boldsymbol{r}, \boldsymbol{r}^{\prime}\right)= & -\frac{1}{4 \pi \cos (\pi \nu)} \frac{\sqrt{\left(r^{2}+\rho^{2}\right)\left(r^{\prime 2}+\rho^{2}\right)}}{\left|\boldsymbol{r}-\boldsymbol{r}^{\prime}\right| \sqrt{r^{2} r^{\prime 2}+2 \rho^{2} \boldsymbol{r} \cdot \boldsymbol{r}^{\prime}+\rho^{4}}} \\
& \times \sin \left[\left(\nu+\frac{1}{2}\right) \arccos \left(-1+\frac{2 \rho^{2}\left(\boldsymbol{r}-\boldsymbol{r}^{\prime}\right)^{2}}{\left(r^{2}+\rho^{2}\right)\left(r^{\prime 2}+\rho^{2}\right)}\right)\right] \quad(N=3) .
\end{aligned}
$$

Equivalence between the result in Eq. (3.48) and the following expression (modified to conform with the present notation and corrected for a sign error):

$$
\begin{aligned}
G_{\nu}\left(\boldsymbol{r}, \boldsymbol{r}^{\prime}\right)= & -\frac{1}{4 \pi \cos (\pi \nu)} \frac{\sqrt{\left(r^{2}+\rho^{2}\right)\left(r^{\prime 2}+\rho^{2}\right)}}{\left|\boldsymbol{r}-\boldsymbol{r}^{\prime}\right| \sqrt{r^{2} r^{\prime 2}+2 \rho^{2} \boldsymbol{r} \cdot \boldsymbol{r}^{\prime}+\rho^{4}}} \\
& \times \sin \left[(2 \nu+1) \arctan \frac{\sqrt{r^{2} r^{\prime 2}+2 \rho^{2} \boldsymbol{r} \cdot \boldsymbol{r}^{\prime}+\rho^{4}}}{\rho\left|\boldsymbol{r}-\boldsymbol{r}^{\prime}\right|}\right] \quad(N=3),
\end{aligned}
$$

given by Demkov and Ostrovsky in Ref. [4], may be easily established with the aid of the well-known inverse trigonometric identity

$$
\arctan \xi=\frac{1}{2} \arccos \frac{1-\xi^{2}}{1+\xi^{2}} \quad(\xi \geqslant 0) .
$$

\section{The generalized Green's function for the fish-eye problem}

A glance at either of Eqs. (3.35), (3.39) or (3.40) reveals that the fish-eye Green's function $G_{\nu}\left(\boldsymbol{r}, \boldsymbol{r}^{\prime}\right)$ fails to exist for the following values of $\nu$ :

$$
\nu=n+N / 2-1 \quad \text { or } \quad \nu=-n-N / 2 \quad(n \in \mathbb{N}),
$$


which, by the way, are solutions of the quadratic equation

$$
\nu(\nu+1)=\left(n+\frac{N}{2}\right)\left(n+\frac{N}{2}-1\right) .
$$

If either of the conditions set in Eq. (4.1) holds, one seeks the generalized Green's function $\bar{G}_{n+N / 2-1}\left(\boldsymbol{r}, \boldsymbol{r}^{\prime}\right) \equiv \bar{G}_{-n-N / 2}\left(\boldsymbol{r}, \boldsymbol{r}^{\prime}\right)$, defined through the limiting relation

$$
\begin{aligned}
\bar{G}_{n+N / 2-1}\left(\boldsymbol{r}, \boldsymbol{r}^{\prime}\right)= & \lim _{\nu(\nu+1) \rightarrow(n+N / 2)(n+N / 2-1)} \\
& \frac{\partial}{\partial[\nu(\nu+1)]}\left\{\left[\nu(\nu+1)-\left(n+\frac{N}{2}\right)\left(n+\frac{N}{2}-1\right)\right] G_{\nu}\left(\boldsymbol{r}, \boldsymbol{r}^{\prime}\right)\right\} \\
= & \frac{1}{2 n+N-1} \lim _{\nu \rightarrow n+N / 2-1} \frac{\partial}{\partial \nu}\left[\left(\nu-n-\frac{N}{2}+1\right)\left(\nu+n+\frac{N}{2}\right) G_{\nu}\left(\boldsymbol{r}, \boldsymbol{r}^{\prime}\right)\right] .
\end{aligned}
$$

If, for instance, representation (3.39) of $G_{\nu}\left(\boldsymbol{r}, \boldsymbol{r}^{\prime}\right)$ is used in Eq. (4.3), this results in

$$
\begin{aligned}
& \bar{G}_{n+N / 2-1}\left(\boldsymbol{r}, \boldsymbol{r}^{\prime}\right)=(-)^{n} \frac{(n+N-2) !}{4 \pi^{N / 2} n !} \frac{\rho^{N / 2-1}}{\left|\boldsymbol{r}-\boldsymbol{r}^{\prime}\right|^{N / 2-1}\left(r^{2} r^{\prime 2}+2 \rho^{2} \boldsymbol{r} \cdot \boldsymbol{r}^{\prime}+\rho^{4}\right)^{N / 4-1 / 2}} \\
& \times\left\{\left[\psi(n+N-1)-\psi(n+1)+\frac{1}{2 n+N-1}\right] P_{n+N / 2-1}^{-N / 2+1}\left(-1+\frac{2 \rho^{2}\left(\boldsymbol{r}-\boldsymbol{r}^{\prime}\right)^{2}}{\left(r^{2}+\rho^{2}\right)\left(r^{\prime 2}+\rho^{2}\right)}\right)\right. \\
& \left.+\left.\frac{\partial P_{\nu}^{-N / 2+1}\left(-1+\frac{2 \rho^{2}\left(\boldsymbol{r}-\boldsymbol{r}^{\prime}\right)^{2}}{\left(r^{2}+\rho^{2}\right)\left(r^{\prime 2}+\rho^{2}\right)}\right)}{\partial \nu}\right|_{\nu=n+N / 2-1}\right\},
\end{aligned}
$$

where

$$
\psi(\zeta)=\frac{1}{\Gamma(\zeta)} \frac{\mathrm{d} \Gamma(\zeta)}{\mathrm{d} \zeta}
$$

is the digamma function. A number of closed-form representations of the derivative $\left[\partial P_{\nu}^{-N / 2+1}(x) / \partial \nu\right]_{\nu=n+N / 2-1}$ required in Eq. (4.4) may be derived from the author's findings for $\left[\partial P_{\nu}^{ \pm m}(z) / \partial \nu\right]_{\nu=n}, z \in \mathbb{C} \backslash(-1,1)$, presented in Refs. [13, 14]; the simplest, and thus potentially most useful, of these expressions are listed in Appendix A.

In the particular case of $N=3$, Eq. (4.4) yields simply

$$
\begin{aligned}
\bar{G}_{n+1 / 2}\left(\boldsymbol{r}, \boldsymbol{r}^{\prime}\right)=\frac{(-)^{n}}{4 \pi^{2}} \frac{\sqrt{\left(r^{2}+\rho^{2}\right)\left(r^{\prime 2}+\rho^{2}\right)}}{\left|\boldsymbol{r}-\boldsymbol{r}^{\prime}\right| \sqrt{r^{2} r^{\prime 2}+2 \rho^{2} \boldsymbol{r} \cdot \boldsymbol{r}^{\prime}+\rho^{4}}} \\
\quad \times\left\{\cos \left[(n+1) \arccos \left(-1+\frac{2 \rho^{2}\left(\boldsymbol{r}-\boldsymbol{r}^{\prime}\right)^{2}}{\left(r^{2}+\rho^{2}\right)\left(r^{\prime 2}+\rho^{2}\right)}\right)\right] \arccos \left(-1+\frac{2 \rho^{2}\left(\boldsymbol{r}-\boldsymbol{r}^{\prime}\right)^{2}}{\left(r^{2}+\rho^{2}\right)\left(r^{\prime 2}+\rho^{2}\right)}\right)\right. \\
\left.+\frac{\sin \left[(n+1) \arccos \left(-1+\frac{2 \rho^{2}\left(\boldsymbol{r}-\boldsymbol{r}^{\prime}\right)^{2}}{\left(r^{2}+\rho^{2}\right)\left(r^{\prime 2}+\rho^{2}\right)}\right)\right]}{2(n+1)}\right\} \quad(N=3) .
\end{aligned}
$$

\section{$5 \quad$ Prospective applications}

The closed-form representations of the fish-eye Green's function $G_{\nu}\left(\boldsymbol{r}, \boldsymbol{r}^{\prime}\right)$ and of its generalized counterpart $\bar{G}_{n+N / 2-1}\left(\boldsymbol{r}, \boldsymbol{r}^{\prime}\right)$, found in this work, are certainly interesting for their own mathematical sake. They appear, however, to be also useful in the physical context. In a forthcoming report, we shall use them to show that, despite of claims to the contrary $[4,8,9]$, in wave optics the infinite Maxwell fish-eye medium does not possess the same perfect focusing properties as it has in geometrical optics. Next, it has been confirmed [15] that the use of either of the closed-form 
expressions for $G_{\nu}\left(\boldsymbol{r}, \boldsymbol{r}^{\prime}\right)$ listed in Section 3 simplifies greatly the mathematical analysis of waveoptical properties of cylindrical $(N=2)$ and spherical $(N=3)$ gradient-index lenses with the fish-eye refraction index (1.1) and of finite radii $r_{\text {lens }} \leqslant \rho \sqrt{2 n_{0}-1}$. Finally, in yet another forthcoming paper, we shall show that there is a close mathematical relationship between the wavized Maxwell fish-eye problem in $\mathbb{R}^{N}$ and the $N$-dimensional Schrödinger-Coulomb problem in momentum space; in particular, we shall provide there an integral expression for the momentum-space Schrödinger-Coulomb Green's function in terms of the fish-eye Green's function discussed above.

\section{A Appendix: The derivatives $\left[\partial P_{\nu}^{-N / 2+1}(x) / \partial \nu\right]_{\nu=n+N / 2-1}$ for $N \in \mathbb{N} \backslash\{0,1\}$}

From the relations

$$
P_{n}^{-m}(x)=\mathrm{e}^{-\mathrm{i} \pi m / 2} \frac{(n-m) !}{(n+m) !} P_{n}^{m}(x+\mathrm{i} 0) \quad(0 \leqslant m \leqslant n)
$$

and

$$
\left.\frac{\partial P_{\nu}^{-m}(x)}{\partial \nu}\right|_{\nu=n}=\left.\mathrm{e}^{-\mathrm{i} \pi m / 2} \frac{(n-m) !}{(n+m) !} \frac{\partial P_{n}^{m}(x+\mathrm{i} 0)}{\partial \nu}\right|_{\nu=n}-[\psi(n+m+1)-\psi(n-m+1)] P_{n}^{-m}(x)
$$

and from a number of closed-form expressions for the derivative $\left[\partial P_{\nu}^{m}(z) / \partial \nu\right]_{\nu=n}$, with $z \in \mathbb{C} \backslash$ $(-1,1)$ and $0 \leqslant m \leqslant n$, found by the present author in Refs. [13, 14], one may derive, among others, the following representations of $\left[\partial P_{\nu}^{-N / 2+1}(x) / \partial \nu\right]_{\nu=n+N / 2-1}$, with $x \in[-1,1]$ and with $N$ being an even natural number greater than zero: ${ }^{1}$

$$
\begin{aligned}
& \left.\frac{\partial P_{\nu}^{-N / 2+1}(x)}{\partial \nu}\right|_{\nu=n+N / 2-1}=P_{n+N / 2-1}^{-N / 2+1}(x) \ln \frac{1+x}{2}-2 \psi(n+N-1) P_{n+N / 2-1}^{-N / 2+1}(x) \\
& +\frac{n !}{(n+N-2) !}\left(\frac{1-x^{2}}{4}\right)^{N / 4-1 / 2} \sum_{k=0}^{n}(-)^{k} \frac{(k+n+N-2) !}{k !(k+N / 2-1) !(n-k) !} \\
& \times[2 \psi(k+n+N-1)-\psi(k+N / 2)]\left(\frac{1-x}{2}\right)^{k} \\
& +\left(\frac{1-x}{1+x}\right)^{N / 4-1 / 2} \sum_{k=0}^{n+N / 2-1}(-)^{k} \frac{(k+n+N / 2-1) ! \psi(k+N / 2)}{k !(k+N / 2-1) !(n+N / 2-k-1) !}\left(\frac{1-x}{2}\right)^{k}
\end{aligned}
$$

$$
\begin{aligned}
& \left.\frac{\partial P_{\nu}^{-N / 2+1}(x)}{\partial \nu}\right|_{\nu=n+N / 2-1}=P_{n+N / 2-1}^{-N / 2+1}(x) \ln \frac{1+x}{2}-2 \psi(n+N / 2) P_{n+N / 2-1}^{-N / 2+1}(x) \\
& +\frac{n !}{(n+N-2) !}\left(\frac{1-x^{2}}{4}\right)^{N / 4-1 / 2} \sum_{k=0}^{n}(-)^{k} \frac{(k+n+N-2) ! \psi(k+N / 2)}{k !(k+N / 2-1) !(n-k) !}\left(\frac{1-x}{2}\right)^{k} \\
& +\left(\frac{1-x}{1+x}\right)^{N / 4-1 / 2} \sum_{k=0}^{n+N / 2-1}(-)^{k} \frac{(k+n+N / 2-1) !}{k !(k+N / 2-1) !(n+N / 2-k-1) !} \\
& \quad \times[2 \psi(k+n+N / 2)-\psi(k+N / 2)]\left(\frac{1-x}{2}\right)^{k},
\end{aligned}
$$

\footnotetext{
${ }^{1}$ Attention! The Mathematica 7.0.0 function LegendreP [nu, mu, 2, x] evaluates incorrectly (the modulus is correct but the sign is wrong) numerical values of the associated Legendre functions $P_{2 n+1}^{-2 n-1}(x)$ and $P_{2 n+2}^{-2 n-1}(x)$ for $n \in \mathbb{N}$, $-1 \leqslant x \leqslant 1$. An empirically discovered remedy is to subject the variable $x$ to the action of the function SetPrecision before it is used as an argument of LegendreP.
} 


$$
\begin{gathered}
\left.\frac{\partial P_{\nu}^{-N / 2+1}(x)}{\partial \nu}\right|_{\nu=n+N / 2-1}=P_{n+N / 2-1}^{-N / 2+1}(x) \ln \frac{1+x}{2} \\
-[\psi(n+N-1)+\psi(n+N / 2)] P_{n+N / 2-1}^{-N / 2+1}(x) \\
+\frac{n !}{(n+N-2) !}\left(\frac{1-x^{2}}{4}\right)^{N / 4-1 / 2} \sum_{k=0}^{n}(-)^{k} \frac{(k+n+N-2) ! \psi(k+n+N-1)}{k !(k+N / 2-1) !(n-k) !}\left(\frac{1-x}{2}\right)^{k} \\
+\left(\frac{1-x}{1+x}\right)^{N / 4-1 / 2} \sum_{k=0}^{n+N / 2-1}(-)^{k} \frac{(k+n+N / 2-1) ! \psi(k+n+N / 2)}{k !(k+N / 2-1) !(n+N / 2-k-1) !}\left(\frac{1-x}{2}\right)^{k}, \quad \text { (A.5) } \\
\left.\frac{\partial P_{\nu}^{-N / 2+1}(x)}{\partial \nu}\right|_{\nu=n+N / 2-1}=P_{n+N / 2-1}^{-N / 2+1}(x) \ln \frac{1+x}{2} \\
\quad-(-)^{n}\left(\frac{1-x^{2}}{4}\right)^{-N / 4+1 / 2} \sum_{k=0}^{N / 2-2} \frac{(k+n) !(N / 2-k-2) !}{k !(n+N-k-2) !}\left(\frac{1+x}{2}\right)^{k} \\
\quad \times[2 \psi(k+n+N / 2)-\psi(k+N / 2)-\psi(k+1)]\left(\frac{1+x}{2}\right)^{k},
\end{gathered}
$$

$$
\begin{aligned}
& \left.\frac{\partial P_{\nu}^{-N / 2+1}(x)}{\partial \nu}\right|_{\nu=n+N / 2-1}=P_{n+N / 2-1}^{-N / 2+1}(x) \ln \frac{1+x}{2} \\
& -[\psi(n+N-1)-\psi(n+1)] P_{n+N / 2-1}^{-N / 2+1}(x) \\
& -(-)^{n} \frac{n !}{(n+N-2) !}\left(\frac{1-x}{1+x}\right)^{N / 4-1 / 2} \sum_{k=0}^{N / 2-2} \frac{(k+n+N / 2-1) !(N / 2-k-2) !}{k !(n+N / 2-k-1) !}\left(\frac{1+x}{2}\right)^{k} \\
& +(-)^{n} \frac{n !}{(n+N-2) !}\left(\frac{1-x^{2}}{4}\right)^{N / 4-1 / 2} \sum_{k=0}^{n}(-)^{k} \frac{(k+n+N-2) !}{k !(k+N / 2-1) !(n-k) !} \\
& \times[2 \psi(k+n+N-1)-\psi(k+N / 2)-\psi(k+1)]\left(\frac{1+x}{2}\right)^{k}, \\
& \left.\frac{\partial P_{\nu}^{-N / 2+1}(x)}{\partial \nu}\right|_{\nu=n+N / 2-1}=P_{n+N / 2-1}^{-N / 2+1}(x) \ln \frac{1+x}{2} \\
& +[\psi(n+1)+\psi(n+N / 2)] P_{n+N / 2-1}^{-N / 2+1}(x) \\
& -(-)^{n} n !(n+N / 2-1) !\left(\frac{1+x}{1-x}\right)^{N / 4-1 / 2}\left(\frac{1-x}{2}\right)^{n+N / 2-1} \\
& \times \sum_{k=1}^{N / 2-1} \frac{(k-1) !}{(k+n) !(k+n+N / 2-1) !(N / 2-k-1) !}\left(\frac{1-x}{1+x}\right)^{k} \\
& -n !(n+N / 2-1) !\left(\frac{1-x}{1+x}\right)^{N / 4-1 / 2}\left(\frac{1+x}{2}\right)^{n+N / 2-1} \\
& \times \sum_{k=0}^{n}(-)^{k} \frac{\psi(n+N / 2-k)+\psi(n-k+1)}{k !(k+N / 2-1) !(n-k) !(n+N / 2-k-1) !}\left(\frac{1-x}{1+x}\right)^{k},
\end{aligned}
$$




$$
\begin{aligned}
\left.\frac{\partial P_{\nu}^{-N / 2+1}(x)}{\partial \nu}\right|_{\nu=n+N / 2-1}=P_{n+N / 2-1}^{-N / 2+1}(x) \ln \frac{1+x}{2} & \left(\frac{1-x}{2}\right)^{n+N / 2-1} \\
+ & {[\psi(n+1)+\psi(n+N / 2)] P_{n+N / 2-1}^{-N / 2+1}(x) } \\
- & (-)^{n} n !(n+N / 2-1) !\left(\frac{1-x}{1+x}\right)^{N / 4-1 / 2} \\
& \times \sum_{k=0}^{N / 2-2} \frac{(N / 2-k-2) !}{k !(n+N / 2-k-1) !(n+N-k-2) !}\left(\frac{1+x}{1-x}\right)^{k} \\
- & (-)^{n} n !(n+N / 2-1) !\left(\frac{1+x}{1-x}\right)^{N / 4-1 / 2}\left(\frac{1-x}{2}\right)^{n+N / 2-1} \\
& \times \sum_{k=0}^{n}(-)^{k} \frac{\psi(k+1)+\psi(k+N / 2)}{k !(k+N / 2-1) !(n-k) !(n+N / 2-k-1) !}\left(\frac{1+x}{1-x}\right)^{k} \\
\left.\frac{\partial P_{\nu}^{-N / 2+1}(x)}{\partial \nu}\right|_{\nu=n+N / 2-1} & P_{n+N / 2-1}^{-N / 2+1}(x) \ln \frac{1+x}{2} \\
+ & {[2 \psi(2 n+N-1)-\psi(n+N-1)-\psi(n+N / 2)] P_{n+N / 2-1}^{-N / 2+1}(x) } \\
+ & \sum_{k=0}^{n-1}(-)^{k+n} \frac{2 k+N-1}{(n-k)(k+n+N-1)}\left[1+\frac{n !(k+N-2) !}{k !(n+N-2) !}\right] P_{k+N / 2-1}^{-N / 2+1}(x) \\
- & \sum_{k=0}^{N / 2-2}(-)^{k+n+N / 2} \frac{2 k+1}{(n+N / 2-k-1)(k+n+N / 2)} P_{k}^{-N / 2+1}(x) .
\end{aligned}
$$

If, in turn, $N$ is an odd natural number greater than 1, then from Eqs. (3.44) and (3.45) one obtains

$$
\begin{aligned}
& \left.\frac{\partial P_{\nu}^{-N / 2+1}(x)}{\partial \nu}\right|_{\nu=n+N / 2-1}=\frac{2}{\pi} Q_{n+N / 2-1}^{-N / 2+1}(x) \arccos x \\
& -\frac{\left(\frac{N-3}{2}\right) !}{2^{N / 2-2} \sqrt{\pi}}\left(1-x^{2}\right)^{-N / 4+1 / 2} \sum_{k=0}^{(N-3) / 2}(-)^{k} \frac{(k+n) !}{k !\left(k+n+\frac{N-1}{2}\right) !\left(\frac{N-3}{2}-k\right) !} \\
& \quad \times\left[\psi\left(k+n+\frac{N+1}{2}\right)-\psi(k+n+1)\right] \sin [(2 k+n+1) \arccos x]
\end{aligned}
$$

and

$$
\begin{gathered}
\left.\frac{\partial P_{\nu}^{-N / 2+1}(x)}{\partial \nu}\right|_{\nu=n+N / 2-1}=\frac{2}{\pi} Q_{n+N / 2-1}^{-N / 2+1}(x) \arccos x+\psi(n+1) P_{n+N / 2-1}^{-N / 2+1}(x) \\
-\sqrt{\frac{2}{\pi} n ! \sum_{k=0}^{(N-3) / 2} \frac{\left(k+\frac{N-3}{2}\right) ! \psi\left(k+n+\frac{N+1}{2}\right)}{2^{k} k !\left(k+n+\frac{N-1}{2}\right) !\left(\frac{N-3}{2}-k\right) !}} \\
\times \frac{\sin \left[\left(k+n+\frac{N-1}{2}\right) \arccos x+\left(k-\frac{N-3}{2}\right) \frac{\pi}{2}\right]}{\left(1-x^{2}\right)^{k / 2+1 / 4}}
\end{gathered}
$$

where

$$
\begin{aligned}
P_{n+N / 2-1}^{-N / 2+1}(x)= & \frac{\left(\frac{N-3}{2}\right) !}{2^{N / 2-2} \sqrt{\pi}}\left(1-x^{2}\right)^{-N / 4+1 / 2} \sum_{k=0}^{(N-3) / 2}(-)^{k} \frac{(k+n) !}{k !\left(k+n+\frac{N-1}{2}\right) !\left(\frac{N-3}{2}-k\right) !} \\
& \times \sin [(2 k+n+1) \arccos x]
\end{aligned}
$$


or, equivalently,

$$
\begin{aligned}
P_{n+N / 2-1}^{-N / 2+1}(x)= & \sqrt{\frac{2}{\pi}} n ! \sum_{k=0}^{(N-3) / 2} \frac{\left(k+\frac{N-3}{2}\right) !}{2^{k} k !\left(k+n+\frac{N-1}{2}\right) !\left(\frac{N-3}{2}-k\right) !} \\
& \times \frac{\sin \left[\left(k+n+\frac{N-1}{2}\right) \arccos x+\left(k-\frac{N-3}{2}\right) \frac{\pi}{2}\right]}{\left(1-x^{2}\right)^{k / 2+1 / 4}}
\end{aligned}
$$

and also

$$
\begin{aligned}
Q_{n+N / 2-1}^{-N / 2+1}(x)= & \frac{\sqrt{\pi}\left(\frac{N-3}{2}\right) !}{2^{N / 2-1}}\left(1-x^{2}\right)^{-N / 4+1 / 2} \sum_{k=0}^{(N-3) / 2}(-)^{k} \frac{(k+n) !}{k !\left(k+n+\frac{N-1}{2}\right) !\left(\frac{N-3}{2}-k\right) !} \\
& \times \cos [(2 k+n+1) \arccos x]
\end{aligned}
$$

or, equivalently,

$$
\begin{aligned}
Q_{n+N / 2-1}^{-N / 2+1}(x)= & \sqrt{\frac{\pi}{2}} n ! \sum_{k=0}^{(N-3) / 2} \frac{\left(k+\frac{N-3}{2}\right) !}{2^{k} k !\left(k+n+\frac{N-1}{2}\right) !\left(\frac{N-3}{2}-k\right) !} \\
& \times \frac{\cos \left[\left(k+n+\frac{N-1}{2}\right) \arccos x+\left(k-\frac{N-3}{2}\right) \frac{\pi}{2}\right]}{\left(1-x^{2}\right)^{k / 2+1 / 4}} .
\end{aligned}
$$

\section{References}

[1] J. Clerk Maxwell, Solutions of problems, Camb. Dublin Math. J. 8 (1854) 188 [reprinted in: The Scientific Papers of James Clerk Maxwell (Cambridge University Press, Cambridge, 1890; Dover, New York, 1965), p. 74], the solution to problem 2.

[2] C. Carathéodory, Über den Zusammenhang der Theorie der absoluten optischen Instrumente mit einem Satze der Variationsrechnung, Sitzungsberichte der Bayerischen Akademie der Wissenschaften. Mathematisch-naturwissenschaftliche Abteilung (1926) 1-18 [reprinted in: C. Carathéodory, Gesammelte mathematische Schriften, Band II (Beck, Munich, 1955), pp. 18197], sections 3 and 4

[3] K. B. Wolf, Geometric Optics on Phase Space (Springer, Berlin, 2004), chapter 6

[4] Yu. N. Demkov, V. N. Ostrovsky, Intrinsic symmetry of the Maxwell 'fish-eye' problem and the Fock group for the hydrogen atom, Zh. Eksp. Teor. Fiz. 60 (1971) 2011 [Sov. Phys. JETP 33 (1971) 1083]

[5] A. Frank, F. Leyvraz, K. B. Wolf, Hidden symmetry and potential group of the Maxwell fish-eye, J. Math. Phys. 31 (1990) 2757

[6] A. Frank, F. Leyvraz, K. B. Wolf, Potential group in optics: the Maxwell fish-eye system, in: Group Theoretical Methods in Physics, V. V. Dodonov, V. I. Man'ko (eds.), Lecture Notes in Physics 382 (Springer, Berlin, 1991), p. 111

[7] A. J. Makowski, K. J. Górska, Quantization of the Maxwell fish-eye problem and the quantumclassical correspondence, Phys. Rev. A 79 (2009) 052116

[8] U. Leonhardt, Perfect imaging without negative refraction, New J. Phys. 11 (2009) 093040

[9] U. Leonhardt, T. G. Philbin, Perfect imaging with positive refraction in three dimensions, preprint arXiv:0911.0552v1

[10] W. Thomson (Lord Kelvin), Reprint of Papers on Electrostatics and Magnetism, Macmillan, London, 1872, Article XIV. 
[11] Yu. N. Demkov, N. V. Semenova, Inversion transformation in the Schrödinger equation, Teor. Mat. Fiz. 60 (1984) 423 [Theor. Math. Phys. 60 (1984) 914]

[12] W. Magnus, F. Oberhettinger, R. P. Soni, Formulas and Theorems for the Special Functions of Mathematical Physics, 3rd ed. (Springer, Berlin, 1966)

[13] R. Szmytkowski, On the derivative of the associated Legendre function of the first kind of integer order with respect to its degree (with applications to the construction of the associated Legendre function of the second kind of integer degree and order), preprint arXiv:0907.3217

[14] R. Szmytkowski, On parameter derivatives of the associated Legendre function of the first kind (with applications to the construction of the associated Legendre function of the second kind of integer degree and order), preprint arXiv:0910.4550

[15] S. Bielski, private communication 